\title{
A NEW OPERATIVE TECHNIQUE FOR AURAL HAEMATOMA IN DOGS: A RETROSPECTIVE CLINICAL STUDY
}

\author{
András GYÖRFFY ${ }^{1,2^{*}}$ and Attila SZIJÁRTÓ ${ }^{1}$ \\ ${ }^{1}$ Semmelweis University, 1st Department of Surgery, Budapest, Hungary; \\ ${ }^{2}$ Veterinary Clinic Tatabánya, Bárdos lakópark u. 2/A, H-2800 Tatabánya, Hungary
}

(Received 10 September 2013; accepted 12 March 2014)

Aural haematoma is treated either by surgical or by conservative treatment. Both techniques usually require several interventions and re-checks. A new operation technique has been evaluated that requires only a single procedure but is as effective as the techniques used previously. The haematoma is opened, cleaned surgically and the two layers of the auricular cartilage are appositioned inside with several suture lines placed on both sides of the incision, alternating the sides approaching to the sagittal cavity. None of the suture lines is stitched through the auricle completely as they all run intradermally on the side of the haematoma and to the cartilage on the other side. Twenty-three dogs with aural haematoma were treated between 2006 and 2012. In 21 (91\%) of them, the haematoma healed without any auricle deformity. No secondary intervention was necessary in any of the cases. None of the 23 animals experienced a relapse during follow-up. The new method offers a minor risk of postoperative complications while accomplishing high healing rates.

Key words: Dog, aural haematoma, suture pattern, ear

Aural haematoma is a frequent disease of the external ear. It is characterised by a purplish rounded hard swelling of the external ear due to blood accumulation in the interstitial space as reported earlier (Kuwahara, 1986a). It is thought to be the result of some traumatic action (Henderson and Horne, 1993; Dávid et al., 2000), but this has not been supported by any direct evidence so far. In some cases, it has been associated with external otitis (Blättler et al., 2007). In most of the cases intradermal eosinophil and mast cell infiltrations were described; these signs are thought to be related to allergic reactions (Joyce and Day, 1997; Cynthia, 2005). Aural haematoma has also been observed in dogs and cats with Otodectes cynotis infection (Kuwahara, 1986b; Harvey et al., 2001), in which case the development of haematoma is suspected to be the result of an immune response to ticks. Since cases have also been reported with no evidence of an infection by ticks, a direct relationship is questionable (Harvey et al., 2001). In summary, the aetiology of the disease is still highly elusive (Dubielzig

*Corresponding author; E-mail: kisallatrendelo@yahoo.com; Phone: 0036 (30) 233-66-91 
et al., 1984; Cynthia, 2005). In the course of the disease serum or blood accumulates in the inner face of the auricle, inside the cartilage and between the cartilage and the skin as reported earlier (Lanz and Wood, 2004; Brown, 2010). In the absence of treatment a chronic callous fibrosis evolves, leading to a cauliflowerlike shrinkage of the pinna (Harvey et al., 2001). The aim of treatment is to remove blood and fibrin debris, to prevent infection, to eliminate the cavity of the haematoma and to inhibit the development of recurrent lesions (Lanz and Wood, 2004; Brown, 2010).

There are several different conservative and surgical methods to treat this disease including opening by operation and closing by pin sutures (Dávid et al., 2000), and its combination with a squeezing sponge (Lanz and Wood, 2004). In this method, after a sagittal opening of the inner auricle, split layers are stabilised by transient sutures performed on each side of the auricle parallel to the ear axis. Haematoma cannot develop between such sandwich-wise squeezed layers. In the course of recovery connective-tissue nodes along the suture inhibit recurrence. In order to avoid deformity of the auricle, a unique sponge is placed on the inner surface of the auricle. In an alternative variant of this method, the stitches are knotted throughout the sponge, giving a more balanced pressure on the entire auricle. Alternatively, the longitudinal incision is performed for the entire length of the haematoma followed by horizontal interrupted mattress sutures placed parallel to the incision. The pinna is dressed with antiseptic gauze, then covered with a compression bandage to keep the ear in an erected position. Mohsin (2010) described that the sutures could be removed 9-10 days postoperatively while bandaging should continue for one week.

Another option is the implantation of a Penrose drain which is placed between the two end-points of the haematoma into the inner sagittal side of the inner auricle. The advantage of this procedure is the extremely short duration of anaesthesia. Both ends of the drain have to be stabilised by sutures as reported by Wilson (1983), while Cynthia (2005) suggested rinsing it every day.

A third option is using a closed suction drainage in which liquids can follow the negative pressure gradient. This can be further utilised by drawing the remaining liquids from the haematoma by a closed suction drainage. A vacuum probe is exchanged 2-3 times a day at the beginning of treatment and less frequently after one week. The drained ear with the suction drainage is fixed to the head. The success rate with closed suction drainage can be as high as $80 \%$ as reported by Swaim and Bradley (1996).

Drainage can also be used without a fixed drain with or without local glucocorticoid injection. In this technique, the haematoma is aspirated every day and the cave is filled up with 1:5 or 1:10 diluted dexamethasone $0.2 \%$ in physiological saline solution. This procedure is repeated until no fluid can be aspirated (approximately 3-5 days) (Kuwahara, 1986a; Romatowski, 1994). Ahirwar et al. (2007) described another draining option that uses a latex drainage. In this case 
local steroids are not used and only single time parenteral and pressure bandage is applied.

Finally, carbon dioxide laser dots can be used to seal the two auricles after a sagittal opening of the haematoma. These incisions stimulate adhesion between the tissue layers. However, a serosanguinous fluid can build up repeatedly. In these cases Dye et al. (2002) recommend a revision surgery.

The methods described above require ordinary postoperative handling including the necessity to treat (and anaesthetise) the animal several times. This can be disadvantageous for large and/or aggressive, hyperactive animals, difficult-to-handle dogs or for working dogs living far from the clinic. In addition, the protection of the treated auricle has to be ensured by using a collar or a bandage for several days. Here, we established a new treatment method for aural haematoma requiring a single intervention only. Our method presented below is a less invasive alternative technique requiring easier post-operative management.

\section{Materials and methods}

In this new method, the animals involved in the study were generally anesthetised and the ear was operated on from its inner surface. In case of external otitis the external ear canal was cleaned first, then the inflammatory exudate was aspirated and inflammatory debris was washed away. Finally, the external ear canal was packed using a cotton swab up to the root of the ear canal to prevent liquids to spill into the operation site. The next step of the surgical technique was similar to previous methods: a longitudinal incision on the ventral surface of the skin of the pinna was made to include the entire length of the haematoma. This incision was generally made in the midline of the pinna. After draining off the accumulated fluid, the cavity was flushed with sterile infusion solution. Placement of several parallel suture lines was carried out across the incision (Fig. 1). A 3/0 monofilament absorbable suture material (Biosyn ${ }^{\mathrm{TM}}$ ) was used for larger breeds, and 4/0 sutures were administered for smaller breeds. In the former case 2 to 3 suture lines, while in the latter case 1 to 2 suture lines were placed on both sides of the incision, alternating the sides approaching to the sagittal cave.

Subsequent deformity of the auricle could be avoided by the use of alternating placement of the suture lines. Breeds with a smaller pinna (e.g. Boxer) were stretched with a cylindrical cushion, thus the longitudinal stitches could be applied more accurately and the risk of deformity was further reduced. None of the sutures was stitched through the auricle completely, they all ran intradermally on the side of the haematoma, and were sutured to the cartilage on the other side (Fig. 2). The linear sectional wound edges were not in direct contact with one another, and the insertion of a suture line pulling the wound tight was omitted. Thus, the wound edges were positioned 1 to $2 \mathrm{~mm}$ from each other at the end of the operation. This was done to enable draining the subsequent regenerative seroma and, thus, to pre- 
vent deformity of the ear or recurrence. Non-steroidal anti-inflammatory drugs were given postoperatively and the auricle was fixed for one day.

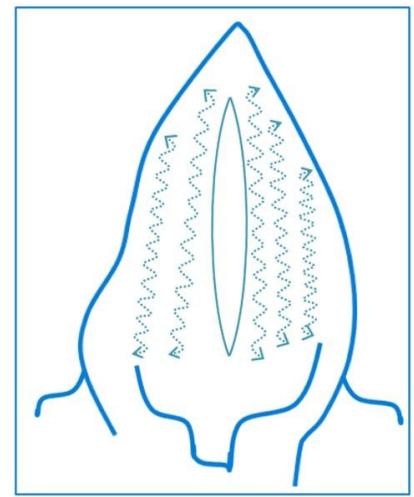

Fig. 1. Following sagittal opening, 1 to 3 suture lines are placed on each side of the incision alternating the sides approaching to the sagittal hole
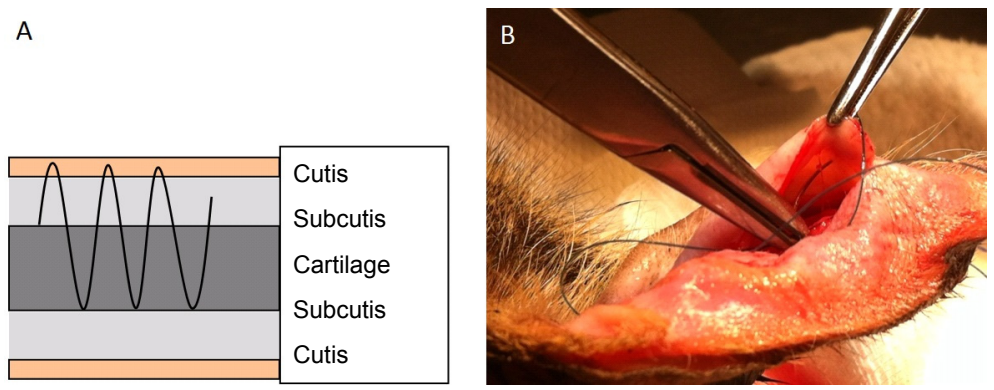

Fig. 2. Suture lines are placed intradermally on one side running through the cartilage on the inner side, as can be seen in schematic view (A) and intraoperative image (B). The skin is not sutured to prevent later bacterial penetration

\section{Results}

Twenty-three dogs were treated in our veterinary clinic between 2006 and 2012. The age of the dogs (12 males and 11 females) was $6 \pm 2.68$ years and ranged between 0.7 and 10 years. The majority of the animals belonged to large breeds: German shepherd $(n=6)$, Rottweiler $(n=3)$, Tyke $(n=4)$; medium-sized dogs were of the German Boxer $(n=3)$ and English cocker spaniel $(n=2)$ breeds. The remaining six dogs were mongrels weighing between 5 and $14 \mathrm{~kg} ; 2$ out of the 6 were Pekinese mixes. Most dogs were middle aged: the youngest animal was 7 months old while the oldest was 10 years old (Fig. 3). 
Twenty-one out of the 23 operated dogs (91\%) healed without any complication (Fig. 4). Additional postoperative management was necessary in $9 \%$ of the cases. For two animals with external otitis the appropriate ear drops had to be given by the owner. In one case ear drops containing polymyxin $\left(\operatorname{Surolan}{ }^{\mathbb{B}}\right.$ ), while in the other case those containing gentamicin (Garasone ${ }^{\circledR}$ ) were used twice a day. Both drops were chosen according to the results of the bacterial susceptibility test performed as part of the routine diagnostic protocols. Auricles of two dogs were finally not re-examined because the owners were unable to transport the dogs into the clinic due to the animals' aggressive behaviour. The owners later reported complete healing of these animals in a telephone interview.

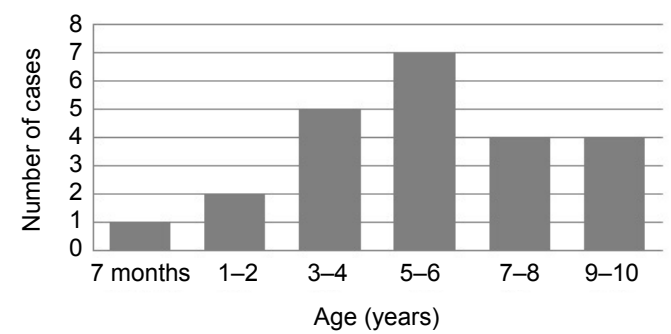

Fig. 3. Distribution of patient age and disease appearance
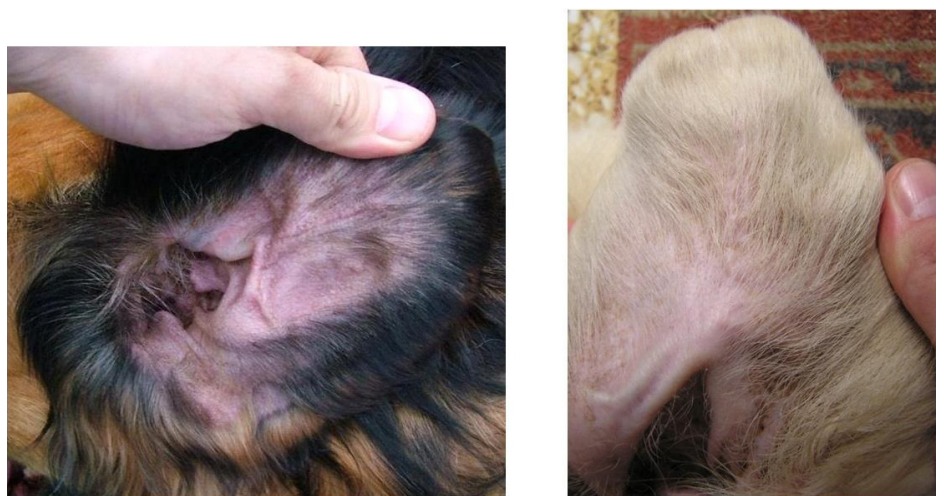

Fig. 4. Postoperative image of the left and right auricle in two dogs subjected to surgery by the new technique. A small sagittal red/white line marks the operating incision two months after surgery

Two dogs had some auricle deformity after the operation (9\%). In the first case, infection caused the development of nodes in the connective tissue and a thickening of auricle layers. We performed daily washing with disinfectant (iodine solution) for a week and application of a tetracycline-containing cream twice a day. Additional medication was not necessary until healing. In the second case, inappropriate positioning of the surgical sutures caused wavy shrinkage of the auricle layers. None of the deformities influenced the animals' hearing ability 
or quality of life. No other complications were observed in the remaining 21 animals subjected to surgery, and, consequently, further postoperative treatment (including swabbing, rinsing, creaming the pinna or other pinna-specific medication) was not necessary.

During the follow-up time, we treated external otitis in 6 operated animals (in two animals twice in the same ear) but aural haematoma did not evolve in any of the cases. For these cases, washing every second day and administration of ear drops three times a day were sufficient to reach complete healing.

\section{Discussion}

Aural haematoma means blood accumulation in the ear inside the cartilage and between the cartilage and the skin (Kuwahara, 1986a). The aim of any operative or conservative therapeutic pathway is to remove the accumulated blood from the pinna and prevent fluid development later on (Lanz and Wood, 2004; Brown, 2010). In this article we present a new surgical method to treat aural haematoma. Advantages of this method include the minimal need for aftercare (including omitting stitch removal and postoperative control), the short-term postoperative cover of the ear and the minimal complication rate. No relapse occurred in any of the cases. Only $9 \%$ of the treated animals developed an auricle deformity after the operation.

An important drawback of certain techniques is the continuous irritation of the ear at the drain insertion sites as reported previously (Wilson, 1983; Lanz and Wood, 2004). Fully penetrating sutures and the sponge can irritate the skin nociceptors of the pinna. Open methods need protection of the head and the auricles in the entire postoperative period (Dávid et al., 2000). Moreover, the skin can become inflamed or ulcerated, causing an itching sensation to the animal. For these reasons, operated animals frequently shake their heads or scratch themselves, and thus aural haematoma is induced in the contralateral auricle as well (Harvey et al., 2001). Long-lasting cover of the auricle by a protective collar can lead to myiasis in the summertime. Elizabethan collar should not be omitted during the short postoperative period with the novel technique either, even if there are no outward stitches. The Penrose drain and the combined glucocorticoid drainage techniques require an everyday cleaning (Kuwahara, 1986b; Romatowski, 1994; Cynthia, 2005). Animals require strict postoperative supervision at the clinic or at home.

Most operative techniques use nonabsorbable sutures, which have to be removed 9-12 days after the operation (Wilson, 1983; Dávid et al., 2000; Lanz and Wood, 2004; Cynthia, 2005; Mohsin, 2010). A further important advantage of the newly described method is that there is no need to remove the stitches. The reason for this is the stable anchorage between layers of the auricle which lasts until the threads have been completely absorbed. During the absorption time of 
monofilament (Biosyn ${ }^{\mathrm{TM}}$ ) sutures taking about 90 days the animal does not feel any discomfort because of the stitches. This means less stress for the dogs. Due to the multiple parallel rows of stitches, layers of the auricle are fixed to each other by several lines. Thus, the probability of recurrence is very low.

A frequent complication of aural haematoma without adequate treatment is the cauliflower-like shrinkage of the auricle (Harvey et al., 2001). In the proposed operative technique this complication can be avoided by accurate fixing. The auricle layers are attached together in accordance with the original location, thereby preventing deformity of the ear. We did not observe either this complication or recurrence of the disease in any case, even if external otitis subsequently occurred in the operated ear. Deformity developed in two cases only, once following a bacterial infection and once due to improperly placed sutures. However, these aesthetic defects did not affect adversely the animals' quality of life. Morphological changes were not the cause of otitis externa or media in any of the cases.

This technique is a viable option for the surgical treatment of aural haematoma in any patient. Nevertheless, it is most useful for a group of animals that are difficult to be repeatedly transported to the veterinary clinic due to either their size and aggressive behaviour or the distance from the veterinary practice.

In summary, in this paper we present a new, less invasive operative technique of aural haematoma for practising surgeons. The utilisation of nonpenetrating absorbable monofilament sutures provides an advantage over previous techniques. The new method requires a lesser amount of postoperative care and leads to a minimal rate of complications.

\section{References}

Ahirwar, V., Chandrapuria, V. P., Bhargava, M. K., Swamy, M., Shahi, A. and Jawre, S. (2007): A comparative study on the surgical management of canine aural haematoma. Indian J. Vet. Surg. 28, 98-100.

Blättler, U., Harlin, O., Mattison, R. G. and Rampelberg, F. (2007): Fibrin sealant as a treatment for canine aural haematoma: a case history. Vet. J. 173, 697-700.

Brown, C. (2010): Surgical management of canine aural hematoma. Lab. Anim. (NY) 39, 104-105.

Cynthia, M. K. (2005): Disease of the Pinna. The Merck Veterinary Manual. 9th edition. Merck Co., Inc. pp. 419-420.

Dávid, T., Kasper, I. and Kasper, M. (2000): Atlas der Kleintierchirurgie. 2nd edition. Schlütersche, Hanover. 213 pp.

Dubielzig, R. R., Wilson, J. W. and Seireg, A. A. (1984): Pathogenesis of canine aural hematomas. J. Am. Vet. Med. Assoc. 185, 873-875.

Dye, T. L., Teague, H. D., Ostwald, D. A. Jr. and Ferreira, S. D. (2002): Evaluation of a technique using the carbon dioxide laser for the treatment of aural hematomas. J. Am. Anim. Hosp. Assoc. 38, 385-390.

Harvey, R. G., Harari, J. and Delauche, A. J. (2001): Ear Diseases of the Dog and Cat. First edition. Wiley-Blackwell, Iowa State University Press, Ames. pp. 226-231.

Henderson, R. A. and Horne, R. D. (1993): The pinna. In: Slatter, D. (ed.) Textbook of Small Animal Surgery. 2nd edition. Saunders, W. B., Philadelphia. pp. 1545-1559. 
Joyce, J. A. and Day, M. J. (1997): Immunopathogenesis of canine aural haematoma. J. Small Anim. Pract. 38, 152-158.

Kuwahara, J. (1986a): Canine and feline aural hematomas: results of treatment with corticosteroids. J. Am. Anim. Hosp. Assoc. 22, 641-647.

Kuwahara, J. (1986b): Canine and feline aural hematoma: clinical, experimental, and clinicopathologic observations. Am. J. Vet. Res. 47, 2300-2308.

Lanz, O. I. and Wood, B. C. (2004): Surgery of the ear and pinna. Vet. Clin. North Am. Small Anim. Pract. 34, 567-599.

Mohsin, A. J. F. (2010): Surgical treatment of ear haematoma in dogs. Bas. J. Vet. Res. 9, 65-70.

Romatowski, J. J. (1994): Nonsurgical treatment of aural hematomas. J. Am. Vet. Med. Assoc. 204, 1318

Swaim, S. F. and Bradley, D. M. (1996): Evaluation of closed-suction drainage for treating auricular hematomas. J. Am. Anim. Hosp. Assoc. 32, 36-43.

Wilson, J. W. (1983): Treatment of auricular hematoma, using a teat tube. J. Am. Vet. Med. Assoc. 182, 1081-1083. 